\title{
TRAPPC11-related muscular dystrophy with hypoglycosylation of alpha-dystroglycan in skeletal muscle and brain
}

\author{
Pinki Munot $^{1}$ | Nadine McCrea ${ }^{1}$ | Silvia Torelli ${ }^{2}$ @ | Adnan Manzur ${ }^{1}$ | \\ Caroline Sewry $^{3}$ | Darren Chambers ${ }^{3}$ | Lucy Feng ${ }^{3}$ | Pierpaolo Ala ${ }^{2}$ | \\ Irina Zaharieva ${ }^{2}$ | Nicola Ragge ${ }^{4}$ | Helen Roper ${ }^{5}$ | Tamas Marton ${ }^{6}$ | Phil Cox ${ }^{6}$ | \\ Miroslav P. Milev ${ }^{7}$ | Wen-Chen Liang ${ }^{8}$ | Shinsuke Maruyama ${ }^{9}$ | Ichizo Nishino ${ }^{10}$ | \\ Michael Sacher ${ }^{7,11}$ | Rahul Phadke ${ }^{3,12}$ ( ) Francesco Muntoni ${ }^{1,13}$ \\ ${ }^{1}$ Dubowitz Neuromuscular Centre, Great Ormond Street Hospital for Children NHS Foundation Trust, London, UK \\ ${ }^{2}$ UCL, Dubowitz Neuromuscular Centre, Great Ormond Street Institute of Child Health, London, UK \\ ${ }^{3}$ Dubowitz Neuromuscular Centre, Division of Neuropathology, UCL Institute of Neurology, London, UK \\ ${ }^{4}$ Birmingham Women's and Children's NHS Foundation Hospital Trust, West Midlands Regional Clinical Genetics Service and Birmingham Health Partners, \\ Birmingham, UK \\ ${ }^{5}$ Department of Paediatrics, Birmingham Heartlands Hospital, University Hospitals Birmingham NHS Foundation Trust, Birmingham, UK \\ ${ }^{6}$ Department of Histopathology, Birmingham Women's and Children's NHS Foundation Trust, Birmingham, UK \\ ${ }^{7}$ Department of Biology, Concordia University, Montreal, Quebec, Canada \\ ${ }^{8}$ Department of Pediatrics, Kaohsiung Medical University Hospital, Kaohsiung, Taiwan \\ ${ }^{9}$ Department of Paediatrics, Kagoshima University Graduate School of Medical and Dental Sciences, Kagoshima, Japan \\ ${ }^{10}$ Department of Neuromuscular Research, National Institute of Neuroscience, National Centre of Neurology and Psychiatry, Kodaira, Japan \\ ${ }^{11}$ Department of Anatomy and Cell Biology, McGill University, Montreal, Quebec, Canada \\ ${ }^{12}$ Division of Neuropathology, University College London Hospitals NHS Foundation Trust National Hospital for Neurology and Neurosurgery, London, UK \\ ${ }^{13}$ UCL Great Ormond Street Institute of Child Health, NIHR Great Ormond Street Hospital Biomedical Research Centre, London, UK
}

\section{Correspondence}

Rahul Phadke, Dubowitz Neuromuscular Centre, Division of Neuropathology, UCL Institute of Neurology, London WC1N 3BG, UK.

Email: r.phadke@ucl.ac.uk

Francesco Muntoni, UCL Great Ormond Street Institute of Child Health, NIHR Great Ormond Street Hospital Biomedical Research Centre, London, WC1N 1EH, UK.

Email: f.muntoni@ucl.ac.uk

\begin{abstract}
Aims: TRAPPC11, a subunit of the transport protein particle (TRAPP) complex, is important for complex integrity and anterograde membrane transport from the endoplasmic reticulum (ER) to the ER-Golgi intermediate compartment. Several individuals with TRAPPC11 mutations have been reported with muscle weakness and other features including brain, liver, skeletal and eye involvement. A detailed analysis of brain and muscle pathology will further our understanding of the presentation and aetiology of TRAPPC11 disease.

Methods: We describe five cases of early-onset TRAPPC11-related muscular dystrophy with a systematic review of muscle pathology in all five individuals, post-mortem brain pathology findings in one and membrane trafficking assays in another.

Results: All affected individuals presented in infancy with muscle weakness, motor delay and elevated serum creatine kinase (CK). Additional features included cataracts, liver disease, intellectual disability, cardiomyopathy, movement disorder and structural brain
\end{abstract}


Funding information

Canadian Institutes of Health Research, Grant/ Award Number: 159645; Health Innovation Challenge Fund, Grant/Award Number: HICF1009-003; Muscular Dystrophy UK; National Institute for Health Research; Natural Sciences and Engineering Research Council of Canada, Grant/Award Number: RGPIN; Seventh Framework Programme, Grant/Award Number: 2012-305121; Wellcome Sanger Institute, Grant/Award Number: WT098051; Department of Health; Wellcome Trust; NHS England Highly Specialised Services; BRC Neuromuscular Centre Biobank; MRC UK; NIHR Great Ormond Street Hospital Biomedical Research Centre abnormalities. Muscle pathology in all five revealed dystrophic changes, universal hypoglycosylation of alpha-dystroglycan and variably reduced dystrophin-associated complex proteins. Membrane trafficking assays showed defective Golgi trafficking in one individual. Neuropathological examination of one individual revealed cerebellar atrophy, granule cell hypoplasia, Purkinje cell (PC) loss, degeneration and dendrite dystrophy, reduced alpha-dystroglycan (IIH6) expression in PC and dentate neurones and absence of neuronal migration defects.

Conclusions: This report suggests that recessive mutations in TRAPPC11 are linked to muscular dystrophies with hypoglycosylation of alpha-dystroglycan. The structural cerebellar involvement that we document for the first time resembles the neuropathology reported in $\mathrm{N}$-linked congenital disorders of glycosylation (CDG) such as PMM2-CDG, suggesting defects in multiple glycosylation pathways in this condition.

\section{KEYWORDS}

cerebellum, dystroglycan, glycosylation, granule cell, IIH6, muscular dystrophy, Purkinje cell, TRAPPC11

\section{INTRODUCTION}

Muscular dystrophies are a heterogeneous group of disorders characterised by progressive muscle weakness with a dystrophic muscle biopsy [1]. Their severity ranges from congenital muscular dystrophy (CMD) with onset within the first year of life to later onset variants, within the spectrum of limb girdle muscular dystrophies (LGMDs) [2]. Multisystem involvement is a feature of several CMDs and LGMDs, particularly with structural brain abnormalities and mental retardation occurring especially in some CMDs, and cardiac involvement coexisting in several CMD and LGMD subtypes. Extracellular matrix proteins and proteins involved in glycosylation of alpha-dystroglycan are frequently involved in CMDs, whereas proteins located in the sarcolemma, members of the dystrophin-associated protein complex (DAPC) and nuclear envelope proteins are the most common proteins involved in LGMDs. Despite significant advances, some individuals remain undiagnosed. Although there has recently been an increase in discovery of novel disease-associated genes by application of whole exome and genome sequencing (WES and WGS), understanding the molecular mechanisms underpinning these novel genes and proteins can be challenging.

In 2013, Bogershausen et al. [3] described three families with an unusual phenotype including muscle weakness and elevated creatine kinase (CK) resembling an LGMD, but with associated dyskinesia, ataxia and intellectual disability. Recessive mutations in the TRAPPC11 gene were identified, leading to the description of LGMD2S. Since then, a few isolated cases affected by either LGMD2S or a more severe congenital form have been reported $[4,5]$. Truncal/limb ataxia and/or hyperkinetic movement disorder is described in a proportion of individuals across the phenotypic spectrum. Mild cerebral atrophy on brain imaging is reported in some individuals, but specific structural brain abnormalities and posterior fossa/cerebellar involvement are not yet described [3-6].

\section{Key points}

- TRAPPC11, is a ubiquitously expressed protein involved in membrane trafficking and in particular anterograde membrane transport from the endoplasmic reticulum (ER) to the ER-Golgi

- We describe recessive TRAPPC11 mutations in patients with muscular dystrophy with reduced glycosylation of alpha dystroglycan (ADG) in skeletal muscle and brain samples.

- Neuropathological examination in one patient reveals structural cerebellar involvement resembling that seen in $\mathrm{N}$-linked congenital disorders of glycosylation (CDG) such as PMM2-CDG, suggesting defects in multiple glycosylation pathways in TRAPPC11-related disease.

Intracellular transport is mediated by vesicles and multi-protein complexes facilitate this process. Transport protein particle (TRAPP) complex is one such complex, and the various component proteins of this complex have a major role in secretory and autophagy pathways including trafficking of various individual and complex proteins (DAPC, sarcoglycan complex, laminin a2, cavins and caveolins) from the endoplasmic reticulum (ER) to the sarcolemma and also from the ER to the Golgi apparatus [7,8]. TRAPPC11, a subunit of the TRAPP complex, is important for complex integrity and anterograde membrane transport from the ER to the ER-Golgi intermediate compartment [9]. Recently a role in autophagy was also suggested [10]. Mutations in other subunits of the TRAPP complex have also been linked with human disease manifesting as a neurodevelopmental disorder with microcephaly, epilepsy and brain atrophy (TRAPPC6B), 
spondyloepiphyseal dysplasia tarda (TRAPPC2), progressive earlyonset encephalopathy with brain atrophy and spasticity (TRAPPC12) and progressive early-onset encephalopathy with episodic rhabdomyolysis (TRAPPC2L) [11-14].

In extensive functional studies on fibroblasts from individuals carrying TRAPPC11 mutations, Bogershausen et al. [3] demonstrated fragmented Golgi, delayed trafficking out of the Golgi and abnormally glycosylated LAMP1 and LAMP2 proteins. The zebrafish model of TRAPPC11-related disease showed impaired $N$-linked glycosylation, with compensatory upregulation in several glycosylation-related genes, including the dystroglycanopathy-related genes GMPPB, DPM1, DPM2 and DPM3 [15]. Matalonga et al. [8] described the first individual with a multisystem CDG with combined defects in $\mathrm{N}$-linked and $O$-linked glycosylation detected in serum proteins (transferrin and apoCIII) and vesicular transport defects in fibroblasts due to TRAPPC11 mutations. In most individuals, compound heterozygous mutations in TRAPPC11 result in quantitative reduction of the full-length TRAPPC11 protein (130 kDa) on immunoblot analysis of muscle or fibroblast lysates, and in some instances, additional lower molecular weight bands are observed suggestive of protein instability, giving rise to truncated TRAPPC11 polypeptide fragments $[3,5,6,8,16]$. Larson et al. [16] described one individual with CMD associated with TRAPPC11 mutations, hypoglycosylation of alpha-dystroglycan in muscle and abnormal membrane trafficking to and from the Golgi in fibroblasts. This study identified a potential novel link between TRAPPC11 and the dystroglycanopathies via the role of TRAPPC11 in the trafficking and glycosylation of dystroglycan in the Golgi. Reduced alpha-dystroglycan immunolabelling in muscle was described in one of two siblings with TRAPPC11-associated LGMD and hepatopathy [17]. However, systematic quantitative studies of alpha-dystroglycan expression in muscle and/or skin from biopsied individuals with TRAPPC11-related disease are lacking. The basis for structural brain involvement in dystroglycanopathies and some common congenital disorders of glycosylation (CDG) such as PMM2-CDG is well characterised with brain imaging and neuropathological studies [18-20]. Although clinical and imaging studies have previously revealed brain involvement in individuals with TRAPPC11 mutations, there is no information to date on its neuropathological basis.

In this study, we describe five cases of muscular dystrophy associated with mutations in TRAPPC11 and discuss a possible role of aberrant glycosylation of alpha-dystroglycan in the disease process with extensive characterisation of muscle pathology and systematic semi-quantitative immunoblot study of alpha-dystroglycan expression in muscle for the first time. We also present the post-mortem findings including neuropathological evaluation of the brain for the first time in one individual and membrane trafficking assays in another. The structural brain defects identified are different from those typically associated in dystroglycanopathies and more closely resemble the cerebellar pathology in defects of $\mathrm{N}$-glycosylation disorders such as PMM2-CDG. Our findings broaden the phenotype and greatly improve our understanding of the pathology of TRAPPC11-associated disorders.

\section{MATERIAL AND METHODS}

All human tissues in this study were acquired and processed under appropriate consent and institutional research ethics cover (REC reference: 13/LO/1894) (NRES Committee London). Whole exome sequence was performed on Patient 5 as part of the Deciphering Developmental Disorders (DDD) Study (Cambridge South REC ref: 10/H0305/83 and Republic of Ireland REC ref: GEN/284/12). Muscle biopsies were processed in accordance with standard protocols as previously described [21]. Semi-quantitative Western blotting on muscle lysates using an antibody against alpha-dystroglycan (IIH6) and laminin overlay assay (LOL) were performed as previously described [22].

Paraffin-embedded samples from the brain were sectioned at $4 \mu \mathrm{m}$ and immunohistochemistry performed on either the Ventana Benchmark Ultra or Discovery Ultra IHC Systems using the standard deparaffinisation protocol and the following antibodies and dilutions: Neurofilament Cocktail (Dako M0762) 1:500, SMI31 (BioLegend 80160) 1:5000, SMI32 (BioLegend 801701) 1:500, SMI94 (BioLegend 836504) 1:100, GFAP (DAKO Z0334) 1:2500, CD68 (Dako M0876) 1:100, ubiquitin (Santa Cruz Biotechnology SC-8017) 1:1000, p62 (BD Bioscience 610833) 1:200, LC3b (Novus Biologicals NB600-1384) 1:800 and TRAPPC11 (Biorbyt ORB186301) 1:200.

Semi-quantitative flow cytometric assay for alpha-dystroglycan was performed on cultured dermal fibroblasts from Individual 1, in accordance with a procedure previously described [23]. Regeneration was assessed qualitatively in sections by identification of fibres expressing fetal/developmental myosin heavy chains (known markers of regeneration). Necrotic fibres were identified by their loss of eosinophilia in H\&E-stained sections with or without myophagocytosis and loss of staining for spectrin (indicating membrane damage).

The trafficking of VSVG-GFP ts045 and quantification of the data was performed in cultured dermal fibroblasts from Individual 1 as described by Koehler et al. [6]. The retention using selective hooks (RUSH) assay was performed and quantified as described by Milev et al. [13].

\section{RESULTS}

\section{Molecular genetics}

WES on the index case (Individual 1) revealed compound heterozygous mutations in TRAPPC11: c.100C > T p. $($ Arg34*) and c.2938G > A p. (Gly980Arg). We further identified four unrelated families with four affected members who all had compound heterozygous mutations as indicated in Table 1.

\section{Clinical features}

Four individuals (Individuals 1, 2, 4 and 5) had onset of symptoms (motor difficulties) in the first year of life, (i.e. a congenital onset), whereas Individual 3 had onset after the age of 1 year. All had 
elevated CK at presentation. Individual 2 has been previously described [5], but here we provide immunoanalysis of alphadystroglycan glycosylation not previously performed.

All individuals acquired independent ambulation except for Individual 5. Cognitive function was impaired in one individual (severely affected infant with CMD, Individual 5). The extra muscular features described in previous TRAPPC11 reports were sought but were not consistently present. Two individuals had bilateral cataracts (Individuals 1 and 2), two had liver involvement (Individuals 2 and 5), and one had a choreiform movement disorder with cerebral and cerebellar atrophy on magnetic resonance imaging (MRI) (Individual 5; Figure 1). This child additionally had cardiomyopathy. Clinical, imaging and laboratory features for all individuals are summarised in Table 1.

\section{Muscle biopsies revealed dystrophic changes and abnormalities in dystrophy-associated proteins in all five individuals}

All five individuals underwent a muscle biopsy taken from either the biceps brachialis or the quadriceps (Figure 2). The age at biopsy ranged from 14 months to 4 years. The biopsies revealed dystrophic changes in all five individuals. These included abnormal size variation, increased internal nuclei, necrosis, regeneration (fetal/developmental myosin positive fibres), fibre splitting, whorling and fibro-fatty infiltration (Individual 1; Figure 2A-E). Immunoanalysis for a broad panel of dystrophy-associated proteins was performed in all individuals (Figure 2). Individuals 1-4 showed moderate, patchy or mosaic reduction in sarcolemmal labelling with an antibody to alphadystroglycan (IIH6, recognising an epitope within the O-glycosylated domain, crucial in binding to laminin alpha-2) (Figure 2G,P,R,T) and subtle reduction in Individual 5 (Figure $2 \mathrm{~W}$ ). Beta-dystroglycan labelling at the sarcolemma appeared normal in Individual 1 (Figure $2 \mathrm{H}$ ). There was variable reduction of several other DAPC proteins including dystrophin and sarcoglycans in two individuals (only Individual 1 illustrated; Figure 2l,J). Spectrin labelling was normal on most of these fibres, indicating an intact sarcolemma (Figure 2F). Unusually, in Individual 1, the sarcolemmal depletion of the DAPC proteins was more pronounced in the larger fibres (Figure $2 \mathrm{I}-\mathrm{K}$ ). These fibres also showed reduced caveolin-3 labelling (Figure $2 \mathrm{~N}$ ). In all cases, a variable but small number of fibres showed cytoplasmic retention of caveolin-3 and/or dysferlin (Figure 2O). Labelling for laminin alpha
FIG URE 1 Brain MRI findings in case 5. MRI of the brain in sagittal (A) and coronal (B) sections demonstrates cerebral and cerebellar atrophy

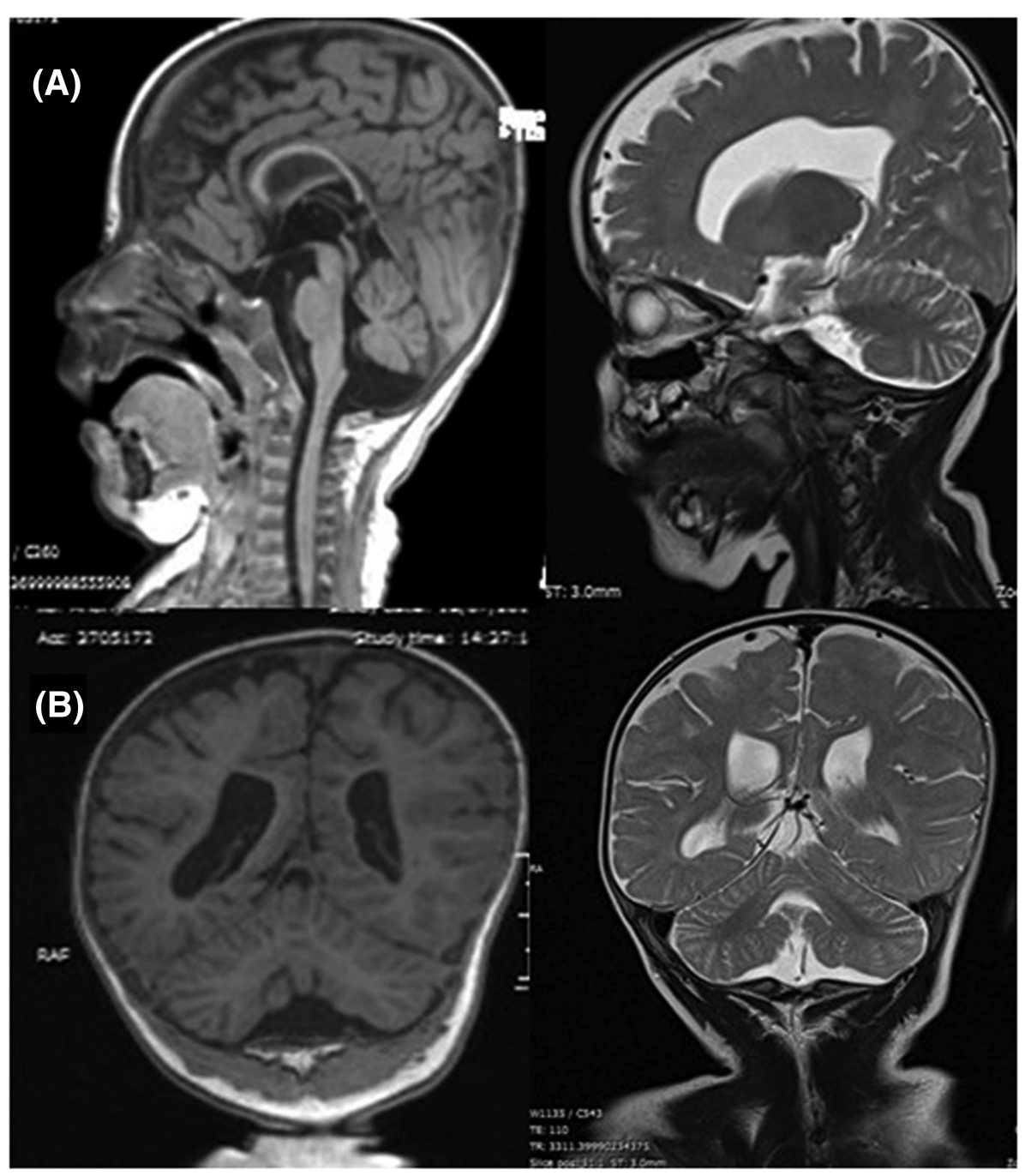



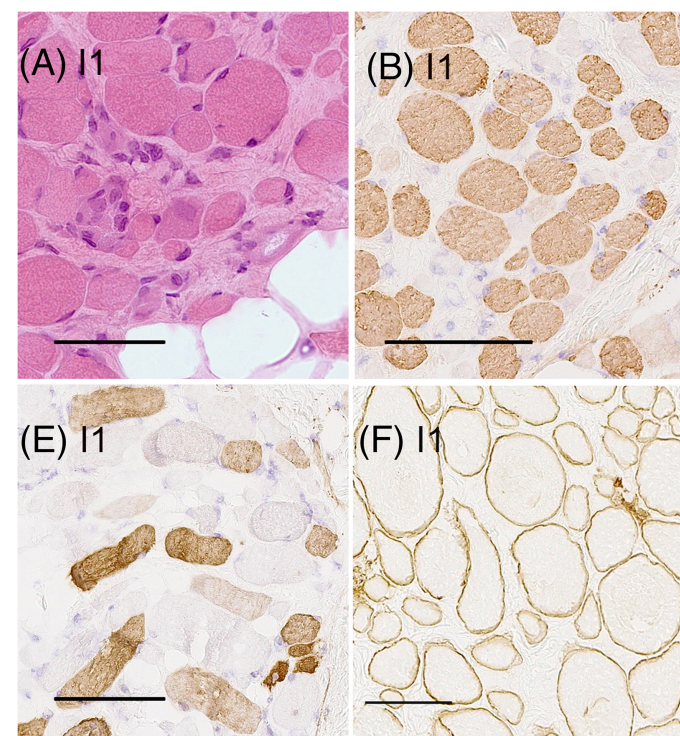

(F) I
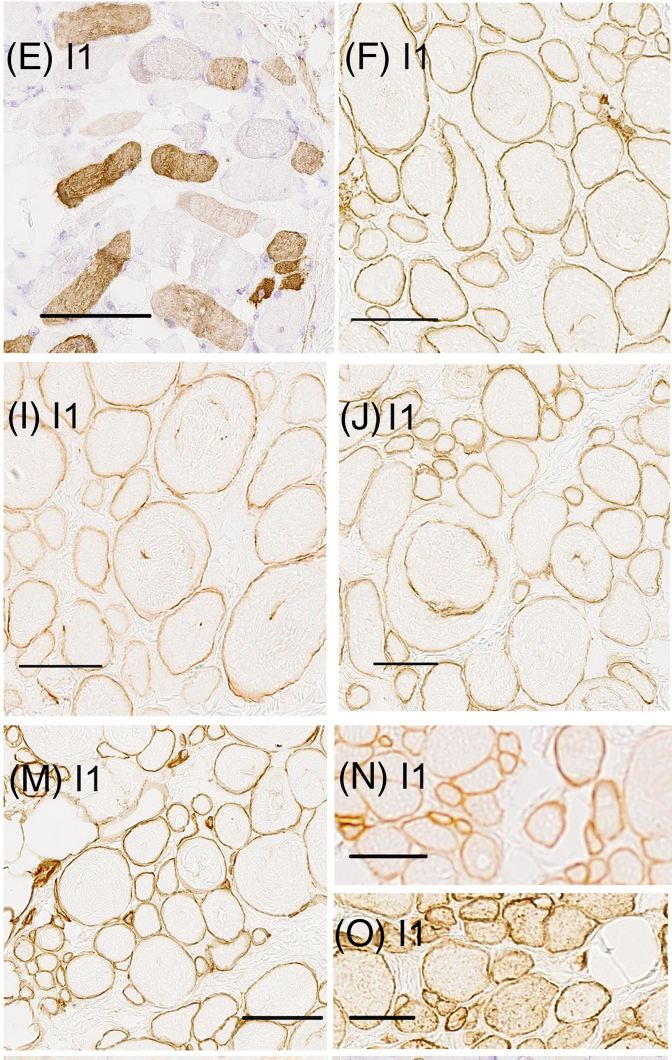

(N) 11

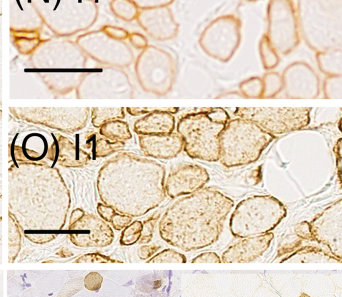

(T) 14

(V) 15

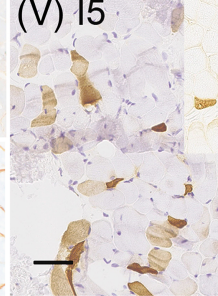

\section{(U) 14}
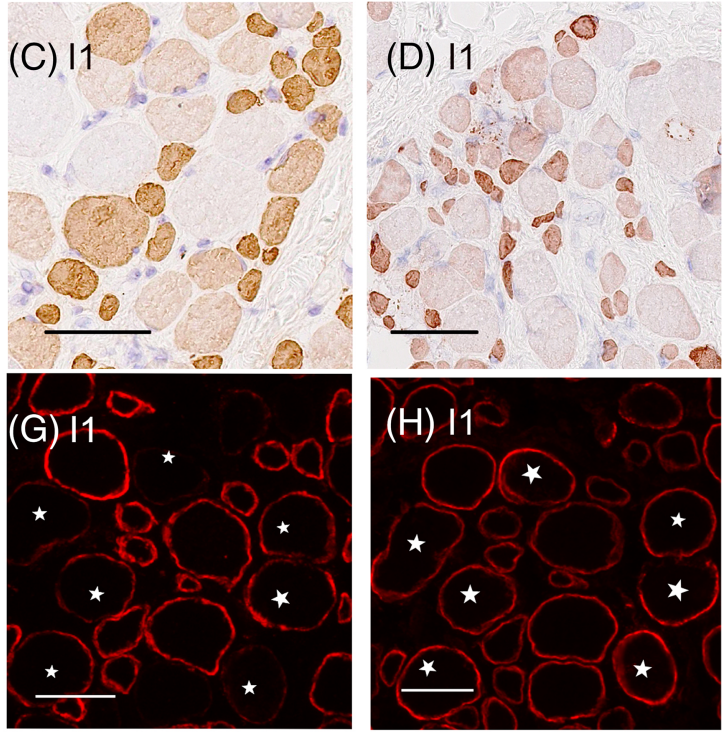

(K) I1

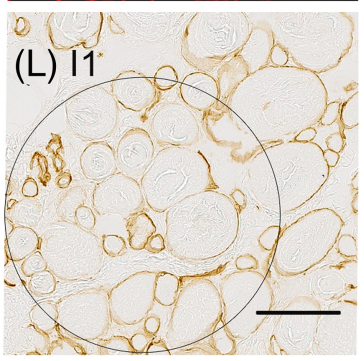

(P) 12

(R) I3
(S) 13

FIG URE 2 Dystrophic muscle pathology from quadriceps and biceps brachialis biopsies. Diagnostic muscle biopsies were performed in all five individuals, from the quadriceps (Individual 1: A-O and 5: V-Y), or the biceps brachialis (Individual 2: P,Q, 3: R,S and 4: T,U). The biopsy from Individual 1 taken at 2 years showed moderate-to-marked dystrophic changes (A). Labelling with slow myosin showed overall slow fibre predominance (B). Fast myosin showed considerable co-expression (C) with preferential expression in smaller fibres clustering around larger preferentially slow myosin expressing fibres. There were many fibres of all sizes and intensities expressing fetal (D) and developmental (E) myosins, consistent with a dystrophic pattern. Most fibres retained robust spectrin labelling indicating an intact sarcolemma (F). There was moderate mosaic/uneven depletion of sarcolemmal alpha-dystroglycan (G), with normal expression of beta-dystroglycan (H). C-terminal dystrophin (I) and alpha-sarcoglycan (J) were patchily reduced, and NNOS (K) was markedly depleted. Laminin alpha 2 (300 kDa) was reduced in a few larger fibres (L) that retained laminin gamma a1 labelling (M), suggesting that the basal lamina was intact in these fibres. There was mild uneven labelling for caveolin-3 $(\mathrm{N})$ and several fibres of varying size showed sarcoplasmic retention of dysferlin (O). Sarcolemmal alphadystroglycan labelling was moderate to severely reduced in Individuals 2-4 (P,R,T). Alpha-sarcoglycan was mildly reduced in Individual 2 (Q) and appeared normal in individuals 3 and $4(\mathrm{~S}, \mathrm{U})$. In Individual 5, the dystrophic changes in the biopsy taken at 14 months were subtle, only recognisable due to a dystrophic labelling pattern for fetal $(\mathrm{V})$ and developmental $(\mathrm{V}$, inset) myosins. Reduction of sarcolemmal alpha-dystroglycan labelling in this individual was subtle (W); labelling for dystrophin and other related proteins including beta-dystroglycan (X), alpha-sarcoglycan (Y) appeared normal. Scale bar: $1 \mathrm{~A}-1 \mathrm{O}, 5 \mathrm{~A}-5 \mathrm{D}=100 \mu \mathrm{m} ; 2 \mathrm{~A}-4 \mathrm{~B}=50 \mu \mathrm{m}$ 
2 (300 and $80 \mathrm{kDa}$ forms) and laminin alpha 5 (excluding regenerating fibres) was normal, except for a few larger fibres with an intact basal lamina (normal labelling for laminin gamma 1, a marker of basal lamina integrity; Figure 2M) showing patchy loss of laminin alpha 2 (Figure 2L). Immunolabelling on sections from histologically normal controls was performed in the same runs as the staining for $\mathrm{I} 1$ and $\mathrm{I} 5$ (Figure S1).

\section{Altered alpha-dystroglycan expression and glycosylation in muscle from all five individuals}

Semi-quantitative Western blotting on muscle lysates using an antibody against alpha-dystroglycan (IIH6) was performed in all cases (Table S1) and showed marked reduction in alpha-dystroglycan expression (17.8\% average intensity) in all five individuals compared with control. This was accompanied by an aberrant expression pattern comprising two to three bands of lower molecular size, strikingly observed in Individual 1 (Figure 3A). Furthermore, a laminin overlay assay revealed a reduction in the glycosylated form, both in intensity (89\% decrease) and in molecular size in this individual (Table S1), represented by a smear on the blot compared with control (Figure 3B). A similar pattern was observed in the remaining individuals showing an average decrease in intensity of $69 \%$ compared with control (Figure S2).

\section{Normal alpha-dystroglycan expression in dermal fibroblasts from Individual 1}

A semi-quantitative flow cytometric assay for alpha-dystroglycan performed on cultured dermal fibroblasts from Individual 1 showed no evidence of reduction in the total number of cells expressing IIH6-reactive glycans ( $91 \pm 0.5$ standard error of mean [SEM] and $98 \pm 0.1$ SEM for control and Individual 1, respectively). Interestingly, we noticed a significant increase (26\%) in IIH6 mean fluorescent signal intensity compared with a control $(813.8 \pm 35$ SEM and $1109 \pm 47$ SEM for control and Individual 1, respectively).

\section{Membrane trafficking into and out of the golgi is delayed in fibroblasts from Individual 1}

Because TRAPPC11 has been implicated in membrane trafficking in the biosynthetic pathway, we examined fibroblasts from control and the affected individual (Individual 1) for the ability to traffic cargo from the ER to and through the Golgi (Figure 4). We first used the RUSH assay [13] to examine the trafficking of two different cargo proteins from the ER to the Golgi. As shown in Figure $4 A, B$, both cargo proteins showed a delay in arrival at the Golgi, consistent with a defect in the early secretory pathway. We also examined the marker VSVG-GFP ts045, a protein that can be conditionally retained in the ER at elevated temperature and released upon

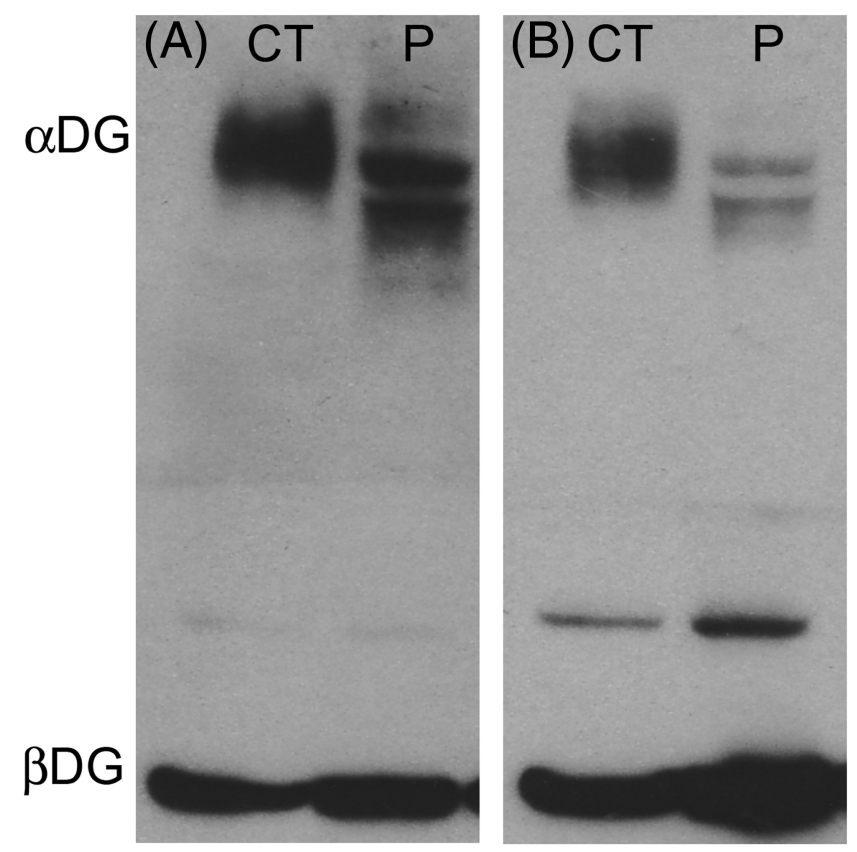

F I G U RE 3 Western blot demonstrates reduced alphadystroglycan glycosylation and laminin binding in muscle. Representative image for Western blot (A) and laminin overlay assay (B) (Individual 1). A reduction in the glycosylated smear of the individual, both in intensity and in molecular weight, was observed compared to the control with antibody IIH6. Three bands are visible in the individual lysate $(A)$ and two of them are also seen with the laminin overlay (B). $\beta$-DG was used as a loading protein. $\alpha$-DG, alphadystroglycan; $\beta$-DG, beta-dystroglycan; $C T$, control; $P$, Individual 1

downshifting the temperature [24]. Similar to the RUSH assay, we noted a delay in the arrival of the fluorescent signal to the Golgi (Figure 4C). In addition, there was a delay in release of the fluorescent signal from the Golgi, suggesting that import into and export from the Golgi are both affected in the presence of the bi-allelic TRAPPC11 variant.

\section{Post-mortem findings in Individual 5}

Individual 5 died at the age of 32 months following cardiovascular complications. A full post-mortem examination was performed. External examination showed right frontal plagiocephaly with a high forehead, downturned mouth and slightly down-slanting eyes with epicanthic folds. The ears were asymmetric. The skin over the neck was loose, and the nipples were widely spaced. There was peripheral oedema. Internal examination revealed moderate ascites and large bilateral pleural effusions. There was moderate cardiomegaly (111.7 g; expected weight for age $74 \mathrm{~g}$ ) and marked left ventricular dilatation, with fibrosis of the left anterior subepicardial myocardium, extending to the interventricular septum. There were no structural cardiac anomalies. The liver was of normal size and weight but appeared pale and fatty. The muscles appeared pale and flabby, with reduced bulk in the chest and calves. The brain was significantly small for his age 


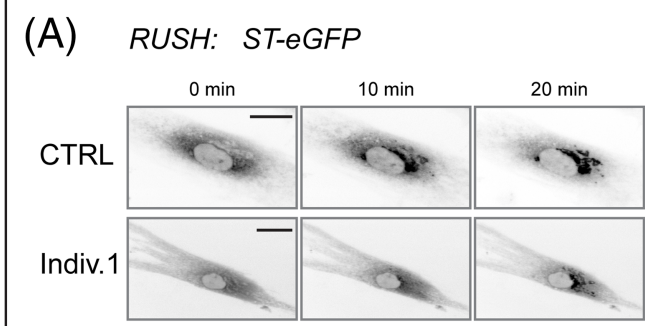

(B) RUSH: Manll-mCherry
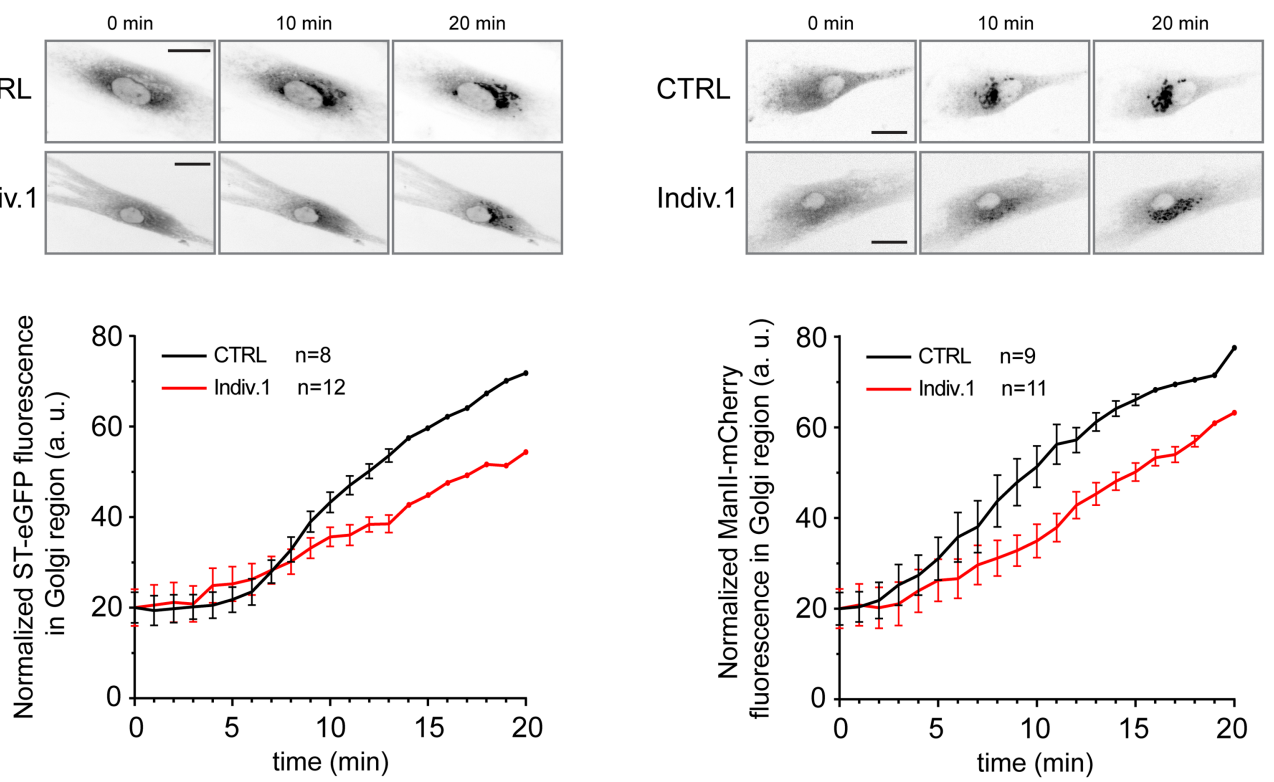

(C) VSVG-GFP ts045
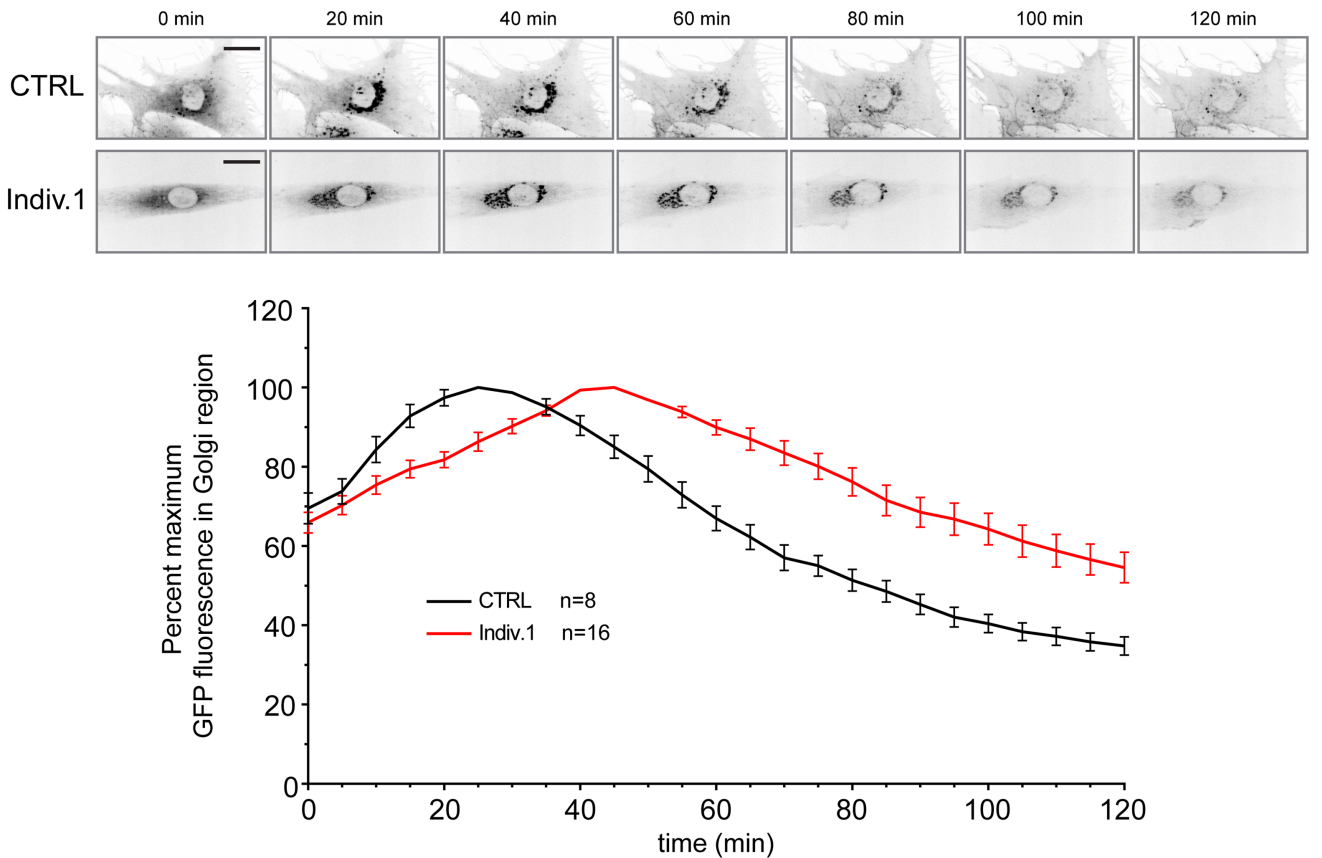

FIG URE 4 Membrane trafficking is delayed in individual 1. Cells from the TRAPPC11 bi-allelic variant display a defect in membrane trafficking. The RUSH assay was performed in fibroblasts from a control and the affected individual (Individual 1) using sialyl transferase (ST)eGFP (A) and mannosidase II (ManlI)-mCherry (B). The signal in the Golgi was quantified as described previously [13] and is plotted beneath the representative images. (C) An assay for the trafficking of VSVG-GFP ts045 into and out of the Golgi of the fibroblasts was performed and quantified as previously described [6]. Error bars represent SEM. Bars in the representative images are $20 \mu \mathrm{m}$

(876 g; expected weight for age is $1120 \mathrm{~g}$ ). The gyral pattern in the forebrain was normal. The cerebellum was atrophic.

Histology revealed mild myocardial hypertrophy with a distinct wideband of myocyte loss and fibrosis in the left ventricular myocardium with septal extension (Figure 5A,B). Minor chronic interstitial inflammation and fatty infiltration in the right ventricle were also noted (data not illustrated). A marked perivenular hepatocyte fatty change and patchy perivenular necrosis associated with bridging 

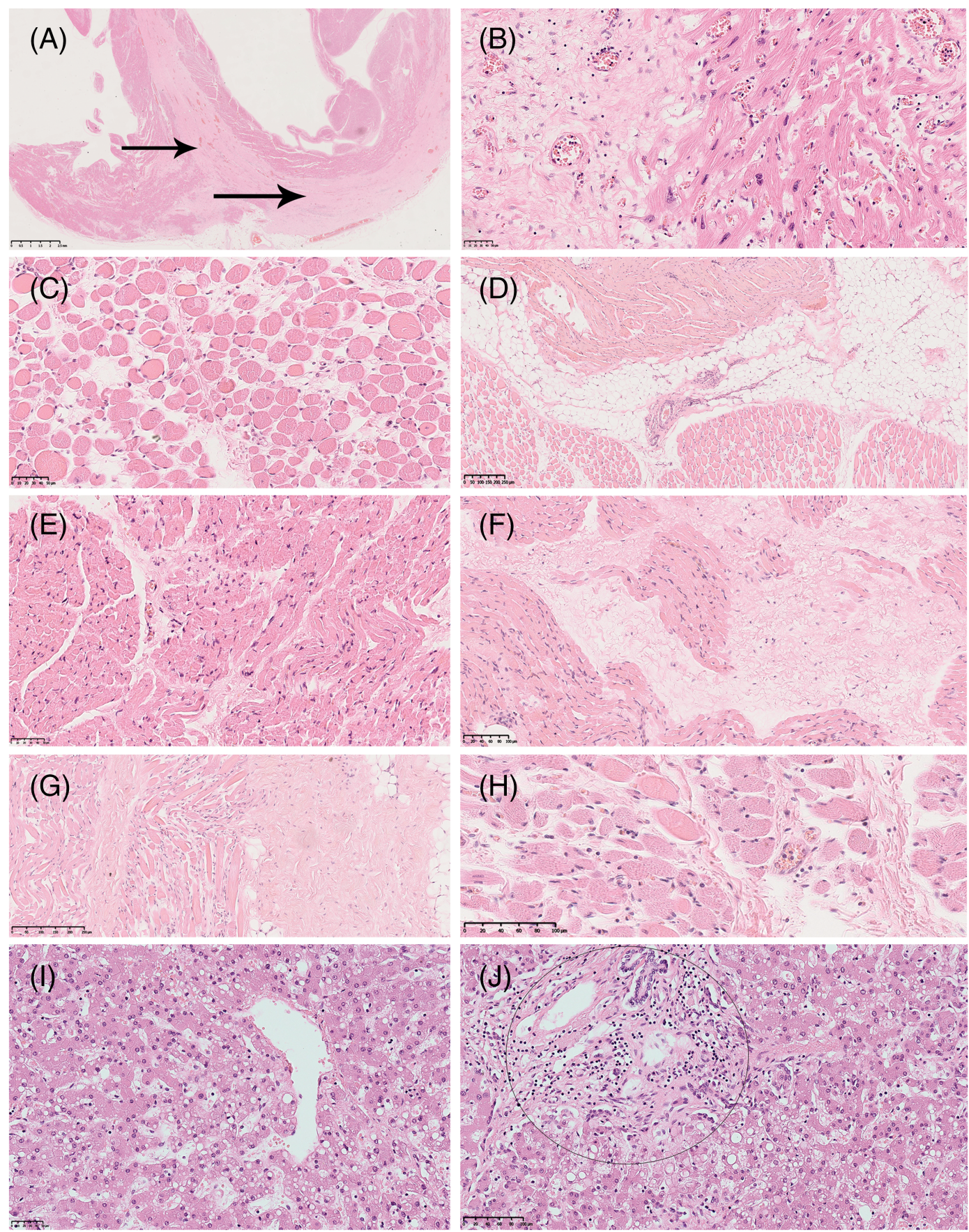

F I G U RE 5 Post-mortem systemic pathology in Individual 5. Post-mortem pathology in Individual 5. Heart (A,B), skeletal muscles (C-H) and liver (I,J). Haematoxylin and eosin-stained section from the left and right ventricle with adjoining septum showed a concentric thick band of fibrosis (arrows) in the outer and mid-myocardium (A). Higher magnification shows an area of scarring adjacent to mildly hypertrophied cardiomyocytes (B). Marked dystrophic changes were seen in multiple skeletal muscles samples including the quadriceps (C,D), calf (E,F), pectoralis $(G)$ and diaphragm $(H)$. There were confluent areas of total fibro-fatty replacement in the quadriceps $(D)$ and calf $(F)$. The liver showed marked perivenous changes including sinusoidal congestion and fatty degeneration of hepatocytes (I) with accompanying portal bridging fibrosis and bile ductular proliferation (circle) (J). Scale bar: $A=1 \mathrm{~mm} ; \mathrm{B}, \mathrm{C}, \mathrm{D}, \mathrm{F}-\mathrm{J}=100 \mu \mathrm{m} ; \mathrm{E}=50 \mu \mathrm{m}$

portal fibrosis and bile ductular proliferation were seen (Figure 5I,J). Dystrophic changes in muscles (quadriceps, calf, pectoralis and diaphragm) including myopathic size variation, internal nucleation, fibrosis and fatty infiltration were noted (Figure $5 \mathrm{C}-\mathrm{H}$ ). In the quadriceps, there was a dramatic progression of the dystrophic changes from the time of the first biopsy at 14 months, with large areas of complete fibro-fatty replacement adjacent to dystrophic areas in the postmortem samples (Figure 5C,D).

Neuropathological examination showed intact cortical organisation in the forebrain. In particular, there was no evidence of neuronal 

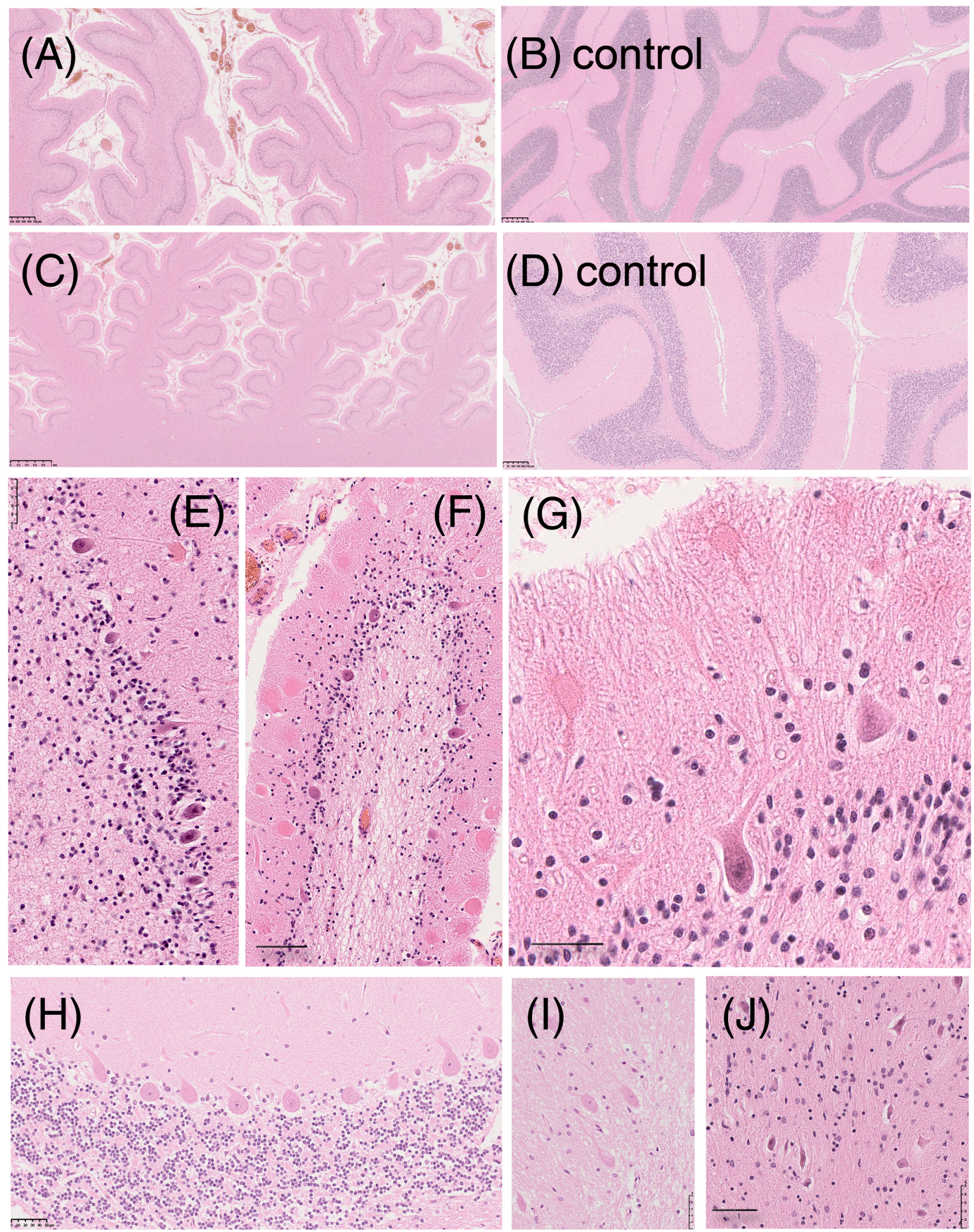

F I G U RE 6 Post-mortem brain pathology in Individual 5: histological features. Haematoxylin and eosin-stained sections showed marked vermian (A) and hemispheric (C) atrophy of the folia and widened inter-folial spaces compared with sections from the vermis (B) and hemisphere (D) from an age-matched control. There was sub-total loss of granule cells and frequent, patchy loss of PC (E,F). Surviving Purkinje cells showed shrunken cell bodies and remarkable dendritic dystrophy, with eosinophilic swellings emanating from proximal or distal dendrites within the molecular layer (E-G). In contrast, the control showed an orderly arrangement of morphologically normal Purkinje cells flanking well-preserved molecular and granule cell layers $(\mathrm{H})$. There was marked shrinkage of neurones within the ribbon of the dentate nucleus and increased cellularity within the neuropil due to microglial activation compared with the control (J). Scale bar: $A, B=500 \mu \mathrm{m}, C=1 \mathrm{~mm}, \mathrm{D}=250 \mu \mathrm{m}, \mathrm{H}=50 \mu \mathrm{m}, \mathrm{E}, \mathrm{F}$, $\mathrm{I}, \mathrm{J}=100 \mu \mathrm{m}, \mathrm{G}=10 \mu \mathrm{m}$

migration defects including cobblestone lissencephaly. There was diffuse neuronal loss, more severe in the outer and middle cortical layers, with neuropil vacuolation. The deep white matter was rarefied, with diffuse cortical and white matter gliosis. The deep grey nuclei were well formed. There was no evidence of brainstem hypoplasia or dysplasia. The ventricles were dilated. The spinal cord appeared normal.

The cerebellum was hypoplastic, with more severe involvement of the vermis. The histological changes were similar in the vermis and 

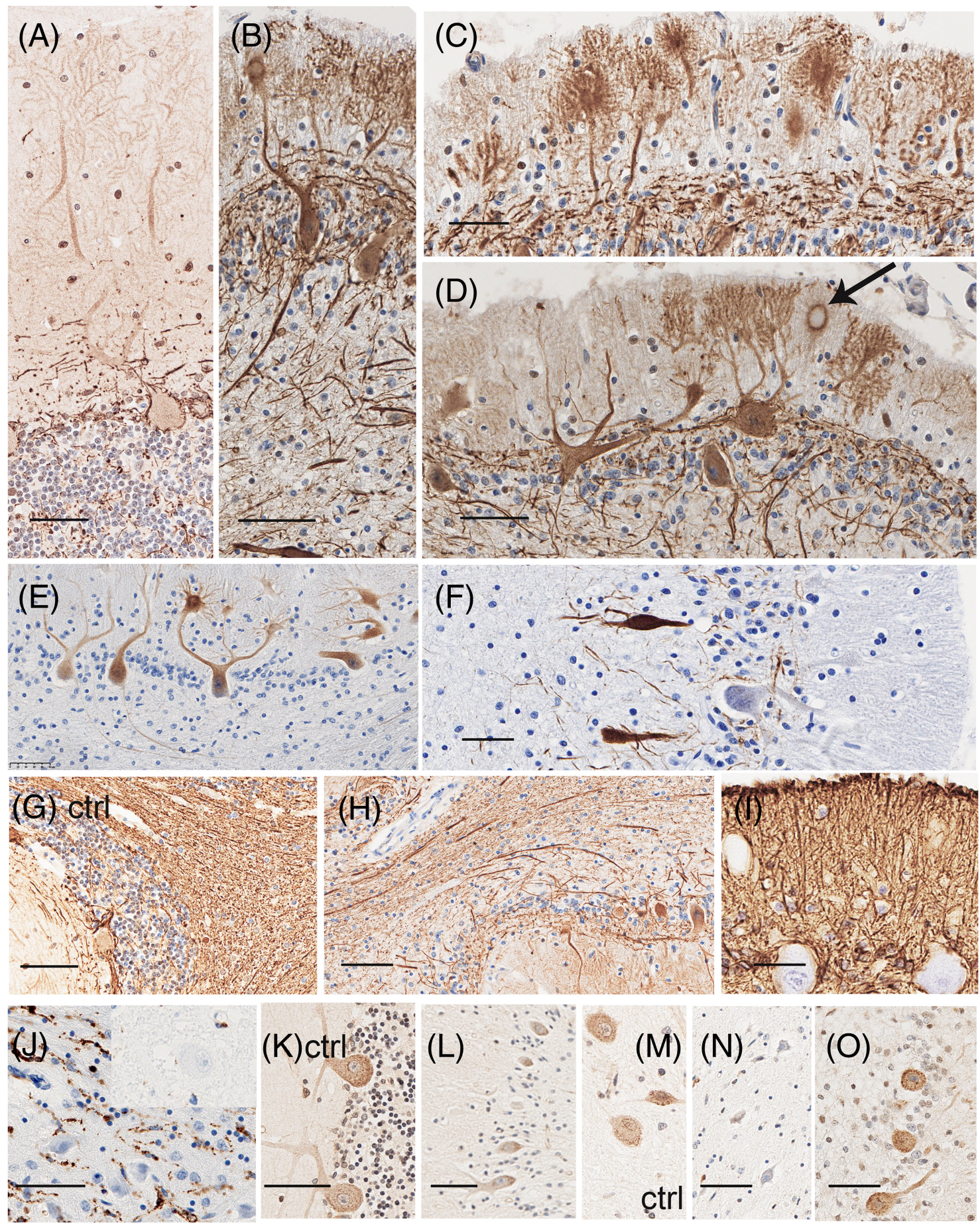

F I G U R E 7 Post-mortem brain pathology in Individual 5: Immunohistochemical characterisation. Neurofilament cocktail (NFC) staining showed an orderly Purkinje cell dendritic arbour in the control (box, A) with robust network of parallel fibres. In the individual, the surviving Purkinje cells showed abnormally oriented dendrites with thickened primary branches and frequent NFC + swellings on terminal and less frequently, proximal primary dendrites with anomalous spines radiating in all directions, so-called asteroid bodies. The dendritic arbour was reduced, and there were patches of the molecular layer with 'disconnected' dendritic arbour with absent Purkinje cell bodies (B-D). A proportion of these swellings displayed immunonegative 'empty cores' (E). Staining with antibodies to non-phosphorylated (SMI32) (F) and phosphorylated (SMI31) (G) neurofilaments showed preferential aggregation of non-phosphorylated neurofilaments within the dendritic swellings. There were scattered Purkinje cell axonal swellings or 'torpedoes' aggregating SMI31 + phosphorylated neurofilaments (G). Compared with the control (H), there was depletion of NFC + axons and rarefaction in the subcortical white matter (I). There was prominent GFAP+ Bergmann/radial gliosis within the molecular layer $(\mathrm{J})$. Brisk CD68 + microglial activation was seen within the shrunken dentate nuclear ribbon (K). IIH6 alphadystroglycan antibody strongly labelled Purkinje cells $(\mathrm{L})$ and dentate neurones $(\mathrm{N})$ in the control. In comparison, IIH6 labelling of Purkinje cells appeared reduced $(\mathrm{M})$, with virtually no labelling of dentate neurones $(\mathrm{O})$ in the individual. There was discernible TRAPPC11 expression in most residual Purkinje cells in the individual (P). Scale bar: $A-O=100 \mu \mathrm{m} ; \mathrm{P}=10 \mu \mathrm{m}$ 
hemispheres. There was diffuse atrophy of the cerebellar folia with widened inter-folial spaces (Figure 6A,C). Granule cells were virtually absent or very severely depleted (Figure 6E,F), with depletion of parallel fibres in the molecular layer. The Purkinje cells (PC) were depleted, and most of the surviving neurones appeared shrunken and showed striking dendritic dystrophy in the form of 'asteroid bodies', which appeared as globular eosinophilic dendritic swellings within the molecular layer (Figure 6E-G). Immunostaining with a high and low molecular weight neurofilament cocktail antibody (NFC) showed a range of abnormalities, including a chaotic and/or dendritic arbour with misdirected, often swollen proximal primary dendrites and dystrophic swellings on primary dendrites seen more often distally, showing anomalous dendritic spines radiating in all directions, corresponding to the 'asteroid bodies' (Figure 7B-D). A proportion of these swellings showed an 'empty core' devoid of any immunoreactivity (Figure 7E). The dendritic swellings were noted to preferentially accumulate SMI32 + non-phosphorylated neurofilaments (Figure 7F,G). The swellings were immunonegative for ubiquitin, p62 and LC3B (markers of autophagy and ubiquitin-proteosomal stress) (data not shown). There were patches of the molecular layer containing a 'disconnected' PC arbour devoid of cell bodies within the depleted PC layer underneath the molecular layer (Figure 7C). There was patchily reduced labelling for alpha-dystroglycan (IIH6) in a proportion of surviving PC (Figure 7M) compared with an age-matched control (Figure $7 \mathrm{~L}$ ), although, in many other cells, the staining appeared indistinguishable from the control. In contrast, there was virtually no labelling for IIH6-reactive glycans in the dentate nucleus (Figure 70) compared with the control (Figure 7N). TRAPPC11 expression was retained in the residual PCs (Figure 7P), dentate neurones, in brainstem nuclei and hippocampal neurones and was comparable with an age-matched control (data not shown). There was atrophy of the dentate grey ribbon, with neuronal shrinkage (Figure 61) and marked activation of CD68 + microglia (Figure 7K). The cerebellar subcortical white matter was rarefied with depletion of NFC + axons (Figure 7l), but there was no evidence of axonal dystrophy or dysmyelination, and there was no cerebellar dysplasia. There was patchy moderate to marked radial gliosis in the molecular layer, particularly in areas of PC loss (Figure 7J).

\section{DISCUSSION}

Dystroglycanopathies are a clinically heterogeneous group of muscular dystrophies, with a wide phenotypic spectrum, from early-onset CMD to milder LGMD presentations, with structural involvement of the brain and eye at the severe end of the spectrum. This is due to defective linkage of the sarcolemma to the extracellular matrix ligands that occurs via the O-linked mannosylated glycan epitope of alphadystroglycan [2]. The molecular genetic background is diverse, most commonly involving mutations in genes encoding glycosyltransferases or enzymes involved in the synthesis of the carbohydrate building blocks. Defects in genes (DOLK, DPM1, DPM2, DPM3, GMPPB) involved in dolichol-P-mannose synthesis result in combined
$\mathrm{N}$-glycosylation and O-mannosylation defects (hypoglycosylation) of alpha-dystroglycan, resulting in dystroglycanopathy [24-27]. Human CDG are clinically heterogeneous diseases usually presenting early in life, often with multiorgan involvement including the brain, muscle, liver and coagulopathy. Defects in several genes encoding enzymes involved in glycosylation pathways or proteins involved in homeostasis of the ER and the Golgi apparatus result in defects of $\mathrm{N}$-glycan assembly (CDG-I), N-glycan processing (CDG-II) or combined defects of $\mathrm{N}$ - and $\mathrm{O}$-glycosylation $[8,28]$.

At least 18 individuals with TRAPPC11 mutations have been reported to date, ranging from the classical LGMD phenotype at the milder end of the spectrum [7], with the clinical course complicated in some instances by scoliosis, or mild intellectual disability and cataracts [3]. At the severe end of the spectrum, individuals with CMD with variable degrees of extra muscular involvement have been described [4,5], including the description of individuals with achalasia, alacrima, steatosis and intellectual disability [6]. A phenotype overlapping CDG Type II was described in a child with neonatal hypotonia, cholestasis, thrombocytopenia, nephropathy, cerebral atrophy and a combined defect of $\mathrm{N}$ - and O-linked glycosylation [8]. Hypoglycosylation of alpha-dystroglycan in muscle has been reported in two individuals with TRAPPC11-muscular dystrophy [16,17].

In the present study, all five individuals underwent a muscle biopsy providing an opportunity for detailed pathology studies. The biopsies revealed dystrophic changes and consistent but variable alpha-dystroglycan hypoglycosylation. Interestingly in Individual 5 , there was a rapid progression of dystrophic changes between the quadriceps biopsy taken at 14 months and following post-mortem examination of the same muscle at 32 months. Immunohistochemical analysis revealed patchy mosaic mild-to-moderate reduction in sarcolemmal labelling for IIH6-reactive glycans on alpha-dystroglycan. Quantitative immunoblotting for alpha-dystroglycan showed severe hypoglycosylation in all cases, accompanied by significant reduction of laminin binding in overlay studies, thereby unequivocally demonstrating glycosylation defects. Conversely, quantitative analysis of alpha-dystroglycan from cultured dermal fibroblasts in Individual 1 did not demonstrate a reduction, but instead, an increase compared with the control. A similar lack of abnormal labelling in fibroblasts was observed by Larson et al. [16] in both their individuals. This may suggest that there are tissue-specific differences in TRAPPC11-mediated Golgi processing and trafficking of alpha-dystroglycan. A similar discrepancy has been reported in other genes implicated in dystroglycanopathies $[29,30]$. The defective traffic into and out of the Golgi seen in our membrane trafficking assays is consistent with what has been noted for other TRAPPC11 variants $[3,9,23]$. In all our cases, we also documented variable, mildly reduced uneven labelling for dystrophin and/or DAPC proteins such as sarcoglycans. This could be secondary to the reduced labelling for alpha-dystroglycan. Sarcolemmal beta-dystroglycan labelling was however well preserved, similar to the findings reported by Larson et al [16] in biopsies from two individuals with compound heterozygous mutations in TRAPPC11. This is not unexpected and is invariably also the case in biopsies from the 'classical' dystroglycanopathies as the interaction of beta- 
dystroglycan with alpha-dystroglycan is independent of its glycosylated epitope [21]. In Individual 1, there was also patchy reduction in sarcolemmal labelling for caveolin-3, and sarcoplasmic retention of caveolin-3 and dysferlin in a number of non-regenerating, nonnecrotic fibres. Caveolin-3 and dysferlin are not part of the DAPC, and sarcolemmal labelling is normally retained in cases with primary or secondary reduction of one or more DAPC proteins. Whereas cytoplasmic retention of caveolin-3 and dysferlin can be observed in regenerating/immature fibres in a variety of dystrophic and/or inflammatory pathologies, the observed cytoplasmic retention in nonregenerating fibres in the biopsy from Individual 1 appears consistent with a wider role for TRAPPC11 in membrane trafficking of proteins (DAPC and non-DAPC) to the sarcolemma and out of the ER in addition to its role in the trafficking and glycosylation of dystroglycan, as well as several serum proteins including transferrin and apoCIII [8].

Although clinical and imaging studies have previously revealed brain involvement in individuals with TRAPPC11 mutations, there was no information on its neuropathological basis. The hallmark of central nervous system involvement in 'classical' alpha-dystroglycanopathies is represented by the cobblestone complex, ranging from complete lissencephaly Type II to more focal pachygyria or polymicrogyria showing frontal predominance. Characteristic infratentorial abnormalities include brainstem and cerebellar dysplasia, hypoplasia and cerebellar cysts. Dystroglycan is expressed in neurones and glia in the developing brain, and radial glia dystroglycan is essential for multiple developmental processes including maintenance of the basement membrane integrity, normal radial glia morphology, organisation of neocortical proliferation and cortical plate lamination [19,31,32].

Neuropathological examination of the brain in Individual 5 with severe CMD revealed notable differences to the structural brain abnormalities associated with the 'classic' dystroglycanopathies. The brain was globally small, but with no evidence of neuronal migration defects in the forebrain. There was marked cerebellar hypoplasia/ cerebellar atrophy $(\mathrm{CA} / \mathrm{CH})$. An absolute distinction between $\mathrm{CA} / \mathrm{CH}$ is difficult in absence of serial imaging [33]. In a large post-mortem series, Divesme et al. [19] analysed 65 fetal and neonatal postmortem cases with histologically confirmed cobblestone lissencephaly. Causal mutations in the six dystroglycanopathy genes known at time (POMT1, POMT2, POMGNT1, LARGE, FKTN and FKRP) were identified in $66 \%$ of cases. The hindbrain was affected in all cases. The cerebellum was grossly reported as being small/malformed/hypoplastic. Histologically, cerebellar dysplasia was reported as focal versus diffuse and major versus minor. This affected both the vermis and hemispheres and featured chaotic organisation and fusion of folia, lacunes comprising a core of arachnoid surrounded by rims of granule cells and/or PC lacking laminar organisation. There were ectopic clusters of granule cells within the arachnoid, fragmented or poorly cellular external granular layer and misoriented PC. Although the cerebellum in Individual 5 in the present study showed grossly similar hypoplasia/ atrophy, histological examination revealed key differences in the underlying pathology. There was virtually complete loss of the granule cells with accompanying Purkinje cell abnormalities including moderate, patchy PC loss and a remarkable dendritic dystrophy affecting surviving Purkinje cells comprising a chaotic and/or dendritic arbour with misdirected, often swollen proximal primary dendrites and dystrophic swellings on primary dendrites seen more often distally, showing anomalous dendritic spines radiating in all directions, corresponding to the 'asteroid bodies'. Morphologically, these changes are strikingly similar to the descriptions of primary degeneration of the granular layer of the cerebellum (Norman type) from the pre-molecular era [34]. Subsequently, it was suggested that the diseases described by Norman and Jaeken are the same pathological entity, with cerebellar pathology identical to that observed in PMM2related CDG. The authors [35] reported seven individuals with severe cerebellar hypoplasia-atrophy, peripheral neuropathy, highly raised serum asialotransferrin and compound heterozygous mutations in PMM2, consistent with PMM2-CDG. Post-mortem examination in one affected individual showed severe loss of granule cells and diverse PC abnormalities including 'cactus-like' or 'asteroid body type' dendritic swellings, identical to the cerebellar pathology reported by Norman and Jaeken. PMM2-CDG is the most frequent $\mathrm{N}$-linked CDG accounting for $80 \%$ of all diagnosed cases. Mutations in PMM2 encoding phosphomannomutase 2 that catalyses conversion of mannose-6-phosphate to mannose-1-phosphate cause the disorder. Although the central and peripheral nervous systems are prime targets, multisystem involvement can occur [36]. PMM2-CDG is a frequent cause of cerebellar ataxia [37]. Most individuals have notable hypoplasia of the superior vermis and cerebellar hemispheres at birth on imaging; milder cases may show initially normal MRI findings, but subsequent progressive atrophy [38]. The neuropathology of PMM2CDG is more extensively characterised among the CDGs. Aronica et al. [20] reported post-mortem findings from an individual with PMM2-CDG (V231M mutation). The structural brain abnormalities were confined to the cerebellum with isolated $\mathrm{CA} / \mathrm{CH}$, granule cell depletion, PC loss, gliosis and abnormal dendritic arborisation and sprouting in the surviving PC. The authors suggest that the occurrence of degeneration following normal migration of $\mathrm{PC}$ rather than developmental arrest during the second and third fetal month is more likely, given the normal placement of surviving PC, occasional axonal swellings and marked cortical gliosis. Antoun et al. reported three children with CDG1; all three had cerebellar atrophy on imaging, with more severe involvement of the anterior lobe. Postmortem examination in one individual revealed complete loss of PC, severely depleted granule cells and gliosis [39]. The authors suggested an antenatal onset based on the vertically oriented tentorium cerebelli in two cases. Taken together, the neuropathological findings in our case closely resemble the striking selective cerebellar involvement in PMM2-CDG, rather than classic dystroglycanopathies. Forebrain structural abnormalities have been reported in other CDGs, for example, sagittal craniosynostosis, hypoplastic corpus callosum and absent septum pellucidum in ALG6CDG, the second most common CDG [40] and partial pachygyria or cobblestone lissencephaly in half of patients with ATP6V0A2-CDG due to combined $\mathrm{N}$ - and O-glycosylation deficiency. These abnormalities are similar but less severe than in the classic dystroglycanopathies [41]. 
Morphological abnormalities of the PC arbour have also been reported in other conditions associated with cerebellar atrophy such as spinocerebellar ataxia type 6, Menkes' disease and familial cortical tremor with epilepsy [20]. In such conditions, there is invariably an associated loss of granule cells, suggesting a response of the PC to partial deafferentation [42]. In our case, few axonal swellings or 'torpedoes' were also noted on residual PC accumulating phosphorylated neurofilaments and prominent Bergmann/radial gliosis in areas of PC loss. Residual cells were however mostly correctly localised to the PC layer. This could suggest normal cellular migration and the occurrence of degeneration rather than developmental arrest during fetal development when PC are generated. The striking neuronal shrinkage in the dentate ribbon with marked microglial activation could also be consequent to loss of the PC input and deafferentation.

Immunostaining revealed the presence of IIH6-reactive glycans in PC, albeit reduced compared with age-matched control, and a virtually total loss of staining in the dentate neurones. Our data provide additional evidence of the overlapping phenotype between dystroglycanopathies and CDG, as previously described in a single case with combined $\mathrm{O}$ - and $\mathrm{N}$-glycosylation defects in a child with neonatal hypotonia, cholestasis, thrombocytopenia, nephropathy and cerebral atrophy [8]. Glycosylation analysis of serum transferrin was normal in this individual (not shown), but analysis of glycoepitopes of secreted proteins may not be sensitive in detection of tissuespecific defects [16]. We could not analyse further $\mathrm{N}$ - and $\mathrm{O}$ glycosylation by quantitative protein assays in the brain tissue of Individual 5 as only formalin-fixed paraffin-embedded material was available.

Granule cells in the cerebellar cortex express dystroglycan during their migration and lose expression during maturation in the internal granule cell layer [43]. In cell-specific conditional dystroglycan-null mice, Nguyen et al. showed that glial dystroglycan, via interaction with the extracellular matrix (ECM) at the glia limitans, is critical for normal cerebellar histogenesis, whereas neuronal dystroglycan is largely unnecessary [44]. It may be tempting to speculate that in patients with TRAPPC11 mutations, there may be temporal and spatial differences in processing of critical glycans including those mediating the ECM interactions of alpha-dystroglycan in the brain compared with other tissues such as skeletal muscle and heart. Residual levels of functional alpha-dystroglycan expressed in critical cell populations such as radial glia may be neuroprotective against neuronal migration defects. There may be yet unidentified TRAPCC11-dependent $\mathrm{N}$ - or O-linked glycans that may be important in the survival of postmigratory cerebellar granule cells.

The present case series further consolidates recessive TRAPPC11 mutations as being causally associated with muscular dystrophies of different clinical severity and hypoglycosylation of alpha-dystroglycan. The CNS shows a lack of the neuronal migration defects of 'classic' alpha-dystroglycanopathies, but instead a distinctive type of cerebellar neurodegeneration resulting in volume loss, severe depletion of granule cells and PC and striking PC dendrite dystrophy, with remarkable similarities to the cerebellar pathology seen in PMM2-CDG. The multisystem involvement with cardiomyopathy and steatohepatosis shows further phenotypic overlap with CDGs. Future work with a focus on animal models and in vitro studies on iPS-derived neuroglial cells derived from patients' fibroblasts to unravel tissue-specific differences in TRAPPC11-mediated processing of $\mathrm{N}$ - and $\mathrm{O}$-linked glycans should be helpful in discerning the complex molecular mechanisms of TRAPPC11-associated disease.

\section{ACKNOWLEDGEMENTS}

We are grateful to the families of the individuals described in this study for extending their support. We acknowledge the financial support from the European Community's Seventh Framework Programme (FP7/2007-2013)-funded grant 'Integrated European -omics research project for diagnosis and therapy in rare neuromuscular and neurodegenerative diseases (NEUROMICS)' (grant agreement number 2012-305121); the Muscular Dystrophy UK Grant on Gene Identification (ref: 18GRO-PG24-0271) to Francesco Muntoni and the NIHR Great Ormond Street Hospital Biomedical Research Centre, UCL GOS Institute of Child Health \& Great Ormond Street Hospital Trust, London, UK. Francesco Muntoni is supported by the National Institute for Health Research Biomedical Research Centre at Great Ormond Street Hospital for Children NHS Foundation Trust and UCL. The support of Muscular Dystrophy UK, MRC UK and BRC Neuromuscular Centre Biobank and NHS England Highly Specialised Services to the Dubowitz Neuromuscular Centre is gratefully acknowledged. The Deciphering Developmental Disorders research study is commissioned by the Health Innovation Challenge Fund (grant number HICF1009-003), a parallel funding partnership between Wellcome Trust and the Department of Health, and the Wellcome Sanger Institute (grant number WT098051). The research team acknowledges the support of the National Institute for Health Research through the Comprehensive Clinical Research Network. Michael Sacher is supported by grants from the Canadian Institutes of Health Research (grant number 159645) and the Natural Sciences and Engineering Research Council of Canada (grant number RGPIN/04385). We acknowledge Mr Lee Martindale, Administrator, DNC Muscle Pathology Service at ION, London, for his administrative assistance. The views expressed are those of the author(s) and not necessarily those of the institutions or funding bodies.

\section{CONFLICT OF INTEREST}

The authors declare that they have no competing interests.

\section{ETHICS STATEMENT}

Informed consent was obtained from the legal guardians of all individuals described in this study. This study was performed in line with the principles of the Declaration of Helsinki. All human tissues in this study were acquired and processed under appropriate consent and institutional research ethics cover (REC reference: 13/LO/1894) (NRES Committee London). Whole exome sequence was performed on Patient 5 as part of the Deciphering Developmental Disorders (DDD) Study (Cambridge South REC ref: 10/H0305/83 and Republic of Ireland REC ref: GEN/284/12). All data depicted in this study 
including radiology and pathology images have been fully anonymised. Further informed consent for publication was obtained from the legal guardians of the individuals described and can be made available.

\section{AUTHOR CONTRIBUTIONS}

Francesco Muntoni, Michael Sacher, Ichizo Nishino, Pinki Munot and Rahul Phadke contributed to the study conception and design. Material preparation, data collection and analysis were performed by Pinki Munot, Nadine McCrea, Silvia Torelli, Adnan Manzur, Caroline Sewry, Pierpaolo Ala, Irina Zaharieva, Nicola Ragge, Helen Roper, Tamas Marton, Phil Cox, Miroslav Milev, Michael Sacher, Wen-Chen Liang, Shinsuke Maruyama, Ichizo Nishino, Rahul Phadke and Francesco Muntoni. Darren Chambers and Lucy Feng provided technical assistance. The first draft of the manuscript was written by Pinki Munot, Nadine McCrea and Rahul Phadke, and all authors commented on previous versions of the manuscript. All authors read and approved the final manuscript.

\section{PEER REVIEW}

The peer review history for this article is available at https://publons. com/publon/10.1111/nan.12771.

\section{DATA AVAILABILITY STATEMENT}

The data that support the findings of this study are available from the corresponding author upon reasonable request.

\section{ORCID}

Silvia Torelli (D) https://orcid.org/0000-0002-8840-6835

Rahul Phadke (D) https://orcid.org/0000-0001-9699-2718

\section{REFERENCES}

1. Laval SH, Bushby KMD. Limb-girdle muscular dystrophies--from genetics to molecular pathology. Neuropathol Appl Neurobiol. 2004; 30(2):91-105.

2. Mercuri E, Bonnemann CG, Muntoni F. Muscular dystrophies. Lancet (London, England). 2019;394(10213):2025-2038.

3. Bogershausen N, Shahrzad N, Chong JX, et al. Recessive TRAPPC11 mutations cause a disease spectrum of limb girdle muscular dystrophy and myopathy with movement disorder and intellectual disability. Am J Hum Genet. 2013;93(1):181-190.

4. Milev MP, Stanga D, Schanzer A, et al. Characterization of three TRAPPC11 variants suggests a critical role for the extreme carboxy terminus of the protein. Sci Rep. 2019;9(1):14036.

5. Liang W-C, Zhu W, Mitsuhashi S, et al. Congenital muscular dystrophy with fatty liver and infantile-onset cataract caused by TRAPPC11 mutations: broadening of the phenotype. Skelet Muscle. 2015;5(1):29.

6. Koehler K, Milev MP, Prematilake K, et al. A novel TRAPPC11 mutation in two Turkish families associated with cerebral atrophy, global retardation, scoliosis, achalasia and alacrima. J Med Genet. 2017; 54(3):176-185.

7. Wang $\mathrm{X}, \mathrm{Wu} \mathrm{Y}$, Cui $\mathrm{Y}$, Wang N, Folkersen L, Wang Y. Novel TRAPPC11 mutations in a Chinese pedigree of limb girdle muscular dystrophy. Case Rep Genet. 2018;2018:8090797.

8. Matalonga L, Bravo M, Serra-Peinado $C$, et al. Mutations in TRAPPC11 are associated with a congenital disorder of glycosylation. Hum Mutat. 2017;38(2):148-151.
9. Scrivens PJ, Noueihed B, Shahrzad N, Hul S, Brunet S, Sacher M. C4orf41 and TTC-15 are mammalian TRAPP components with a role at an early stage in ER-to-Golgi trafficking. Mol Biol Cell. 2011;22(12): 2083-2093.

10. Stanga D, Zhao Q, Milev MP, Saint-Dic D, Jimenez-Mallebrera C, Sacher M. TRAPPC11 functions in autophagy by recruiting ATG2B-WIPI4/WDR45 to preautophagosomal membranes. Traffic (Copenhagen, Denmark). 2019;20(5):325-345.

11. Marin-Valencia I, Novarino G, Johansen A, et al. A homozygous founder mutation in TRAPPC6B associates with a neurodevelopmental disorder characterised by microcephaly, epilepsy and autistic features. J Med Genet. 2018;55(1):48-54.

12. Tiller GE, Hannig VL, Dozier D, et al. A recurrent RNA-splicing mutation in the SEDL gene causes X-linked spondyloepiphyseal dysplasia tarda. Am J Hum Genet. 2001;68(6):1398-1407.

13. Milev MP, Grout ME, Saint-Dic D, et al. Mutations in TRAPPC12 manifest in progressive childhood encephalopathy and Golgi dysfunction. Am J Hum Genet. 2017;101(2):291-299.

14. Milev MP, Graziano C, Karall D, et al. Bi-allelic mutations in TRAPPC2L result in a neurodevelopmental disorder and have an impact on RAB11 in fibroblasts. J Med Genet. 2018;55(11):753-764.

15. DeRossi C, Vacaru A, Rafiq R, et al. trappc11 is required for protein glycosylation in zebrafish and humans. Mol Biol Cell. 2016;27(8): 1220-1234.

16. Larson AA, Baker PR 2nd, Milev MP, et al. TRAPPC11 and GOSR2 mutations associate with hypoglycosylation of alpha-dystroglycan and muscular dystrophy. Skelet Muscle. 2018;8(1):17.

17. Fee DB, Harmelink M, Monrad P, Pyzik E. Siblings with mutations in TRAPPC11 presenting with limb-girdle muscular dystrophy 2S. J Clin Neuromuscul Dis. 2017;19(1):27-30.

18. Angelini C, Pinzan E. Advances in imaging of brain abnormalities in neuromuscular disease. Ther Adv Neurol Disord. 2019;12: 1756286419845567.

19. Devisme L, Bouchet C, Gonzales M, et al. Cobblestone lissencephaly: neuropathological subtypes and correlations with genes of dystroglycanopathies. Brain: J Neurol. 2012;135(Pt 2):469-482.

20. Aronica E, van Kempen AAMW, van der Heide M, et al. Congenital disorder of glycosylation type la: a clinicopathological report of a newborn infant with cerebellar pathology. Acta Neuropathol. 2005; 109(4):433-442.

21. Dubowitz VOA, Sewry CA. Muscle Biopsy E-Book: A Practical Approach. Fifthed. Elsevier; 2020.

22. Brockington M, Torelli S, Sharp PS, et al. Transgenic overexpression of LARGE induces alpha-dystroglycan hyperglycosylation in skeletal and cardiac muscle. PLoS ONE. 2010;5(12):e14434.

23. Stevens E, Torelli S, Feng L, et al. Flow cytometry for the analysis of $\alpha$-dystroglycan glycosylation in fibroblasts from patients with dystroglycanopathies. PLOS ONE. 2013;8(7):e68958.

24. Lefeber DJ, de Brouwer APM, Morava E, et al. Autosomal recessive dilated cardiomyopathy due to DOLK mutations results from abnormal dystroglycan O-mannosylation. PLoS Genet. 2011;7(12): e1002427.

25. Yang AC, Ng BG, Moore SA, et al. Congenital disorder of glycosylation due to DPM1 mutations presenting with dystroglycanopathytype congenital muscular dystrophy. Mol Genet Metab. 2013;110(3): 345-351.

26. Lefeber DJ, Schonberger J, Morava E, et al. Deficiency of Dol-P-Man synthase subunit DPM3 bridges the congenital disorders of glycosylation with the dystroglycanopathies. Am J Hum Genet. 2009;85(1): 76-86.

27. Raphael AR, Couthouis J, Sakamuri S, et al. Congenital muscular dystrophy and generalized epilepsy caused by GMPPB mutations. Brain Res. 2014;1575:66-71.

28. Chang IJ, He M, Lam CT. Congenital disorders of glycosylation. Ann Transl Med. 2018;6(24):477. 
29. Jensen BS, Willer T, Saade DN, et al. GMPPB-associated dystroglycanopathy: Emerging common variants with phenotype correlation. Hum Mutat. 2015;36(12):1159-1163.

30. Carss KJ, Stevens E, Foley AR, et al. Mutations in GDP-mannose pyrophosphorylase $B$ cause congenital and limb-girdle muscular dystrophies associated with hypoglycosylation of alpha-dystroglycan. Am J Hum Genet. 2013;93(1):29-41.

31. Bonnemann CG, Wang CH, Quijano-Roy S, et al. Diagnostic approach to the congenital muscular dystrophies. Neuromuscul Disord: NMD. 2014;24(4):289-311.

32. Myshrall TD, Moore SA, Ostendorf AP, et al. Dystroglycan on radial glia end feet is required for pial basement membrane integrity and columnar organization of the developing cerebral cortex. J Neuropathol Exp Neurol. 2012;71(12):1047-1063.

33. Poretti A, Boltshauser E. Terminology in morphological anomalies of the cerebellum does matter. Cerebellum Ataxias. 2015;2(1):8.

34. Ferrer I, Sirvent J, Manresa JM, Galofre E, Fernandez-Alvarez E, Pineda M. Primary degeneration of the granular layer of the cerebellum (Norman type). A Golgi Study Acta Neuropathol. 1987;75(2): 203-208.

35. Pascual-Castroviejo I, Pascual-Pascual SI, Quijano-Roy S, et al. Cerebellar ataxia of Norman-Jaeken. Presentation of seven Spanish patients. Rev Neurol. 2006;42(12):723-728.

36. Freeze HH, Eklund EA, Ng BG, Patterson MC. Neurology of inherited glycosylation disorders. Lancet Neurol. 2012;11(5):453-466.

37. Boddaert N, Desguerre I, Bahi-Buisson N, et al. Posterior fossa imaging in 158 children with ataxia. J Neuroradiol. 2010;37(4):220-230.

38. Mader I, Dobler-Neumann M, Kuker W, Stibler H, Krageloh-Mann I. Congenital disorder of glycosylation type la: benign clinical course in a new genetic variant. Childs Nerv Syst. 2002;18(1-2):77-80.

39. Antoun H, Villeneuve N, Gelot A, Panisset S, Adamsbaum C. Cerebellar atrophy: an important feature of carbohydrate deficient glycoprotein syndrome type 1. Pediatr Radiol. 1999;29(3):194-198.
40. Eklund EA, Sun L, Yang SP, Pasion RM, Thorland EC, Freeze HH. Congenital disorder of glycosylation Ic due to a de novo deletion and an hALG-6 mutation. Biochem Biophys Res Commun. 2006;339(3): 755-760.

41. Morava E, Lefeber DJ, Urban Z, et al. Defining the phenotype in an autosomal recessive cutis laxa syndrome with a combined congenital defect of glycosylation. Eur J Hum Genet. 2008;16(1):28-35.

42. Urich H. The plasticity of the Purkinje cell. Clin Exp Neurol. 1984;20: 203-215.

43. Henion TR, Qu Q, Smith Fl. Expression of dystroglycan, fukutin and POMGnT1 during mouse cerebellar development. Brain Res Mol Brain Res. 2003;112(1-2):177-181.

44. Nguyen H, Ostendorf AP, Satz JS, et al. Glial scaffold required for cerebellar granule cell migration is dependent on dystroglycan function as a receptor for basement membrane proteins. Acta Neuropathol Commun. 2013;1(1):58.

\section{SUPPORTING INFORMATION}

Additional supporting information may be found in the online version of the article at the publisher's website.

How to cite this article: Munot P, McCrea N, Torelli S, et al. TRAPPC11-related muscular dystrophy with hypoglycosylation of alpha-dystroglycan in skeletal muscle and brain. Neuropathol Appl Neurobiol. 2022;48(2):e12771. doi:10.1111/nan.12771 